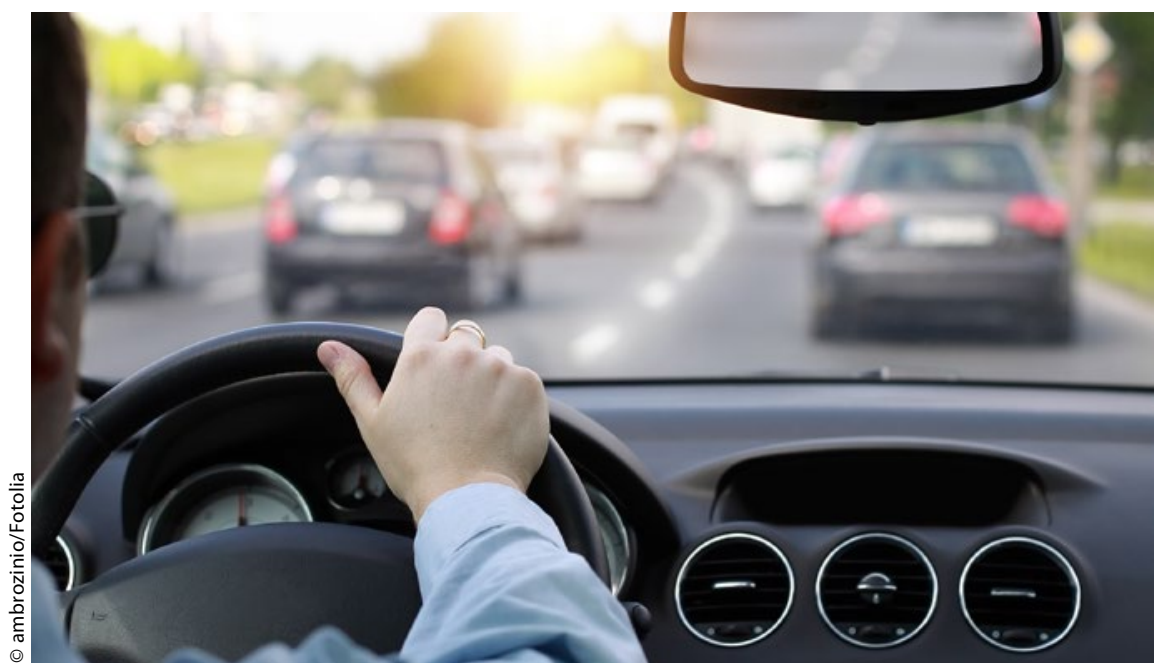

Fahrtüchtigkeit

\title{
Cannabispatienten dürfen Auto fahren
}

- Cannabispatienten dürfen am Straßenverkehr teilnehmen, sofern sie aufgrund der Medikation nicht in ihrer Fahrtüchtigkeit eingeschränkt sind. Die Patienten müssten in der Lage sein, das Fahrzeug "sicher zu führen", heißt es in der Antwort der Bundesregierung auf eine Kleine Anfrage der Fraktion „Die Linke“. Patienten drohe keine Sanktion nach dem Straßenverkehrsgesetz, wenn Cannabis aus der bestimmungsgemäßen Einnahme eines für einen konkreten Krankheitsfall verschriebenen Arzneimittels herrührte. Eine Entziehung der Fahrerlaubnis sei jedoch bei missbräuchlicher Einnahme eines cannabishaltigen Medikaments möglich. Denn die Fahrtüchtigkeit könne auch in der Einstellungs- und Eingewöhnungsphase von cannabishaltigen Arzneimitteln beeinträchtigt sein.
Für die derzeit rund 1.000 Cannabispatienten gelte eine Ausnahmeklausel des Straßenverkehrsgesetzes. Zweck der Regelung sei, dass "durch die Medikation die grundsätzliche Fahrtüchtigkeit erst wieder hergestellt wird“. Die Wirkung der Substanz als Therapeutikum unterscheide sich deutlich von der bei missbräuchlichem Konsum. Patienten seien anders als Drogenkonsumenten sehr zuverlässig und verantwortlich und verhielten sich regelkonform. Gesetzlich sei zwar nicht fest geschrieben, dass Patienten unter Dauermedikation einen Nachweis mitführen müssten. Cannabispatienten werde jedoch empfohlen, beim Führen eines Fahrzeugs eine Ausfertigung des Betäubungsmittelrezeptes oder eine Bescheinigung des Arztes mitzunehmen.

(eb)

\section{Großbritannien \\ Behandlungsstopp für todkrankes Baby angeordnet}

- Ein Gericht in Großbritannien hat gegen den Willen der Eltern einen Behandlungsstopp für ein todkrankes Baby angeordnet, das an mitochondrialer Myopathie leidet und zuletzt künstlich beatmet werden musste. Das Gericht folgte damit einem Antrag der Ärzte des Great-Ormond-Street-Krankenhauses in London. Der acht Monate alte Junge solle in Würde sterben können, begründete der Vorsitzende Richter des High Courts die Entscheidung.

Die Ärzte hatten argumentiert, das Baby könne bei einer weiteren Behandlung möglicherweise Schmerzen erleiden. Durch bereits erlittene Hirnschäden werde sich sein Zustand selbst bei erfolgreicher Behandlung nicht mehr verbessern. Die Eltern hatten dafür gekämpft, ihren Sohn für eine weitere Behandlung in die USA bringen zu können, wofür Sie durch Spenden bereits umgerechnet etwa 1,4 Millionen Euro gesammelt hatten. Sie kündigten an, in Berufung gehen zu wollen.

\section{Nicht-spezifischer Kreuzschmerz \\ Nationale Versorgungsleitlinie aktualisiert}

— Die komplett überarbeitete Nationale VersorgungsLeitlinie (NVL) „Nicht-spezifischer Kreuzschmerz" ist auf den Internetseiten des Ärztlichen Zentrums für Qualität in der Medizin (ÄZQ) kostenlos abrufbar (www.leitlinien.de/nvl/kreuzschmerz). Die NVL enthält 90 Empfehlungen zu Diagnostik, Therapie und Prävention von nicht-spezifischen Kreuzschmerzen und erhebt den Anspruch, den aktuellen Wissensstand für alle an der Versorgung Beteiligten zusammenzufassen. Das Programm für Nationale VersorgungsLeitlinien steht unter der Trägerschaft von Bundesärztekammer, Kassenärztlicher Bundesvereinigung und der Arbeitsgemeinschaft der Wissenschaftlichen Medizinischen Fachgesellschaften. Mit der Durchführung wurde das ÄZQ beauftragt.

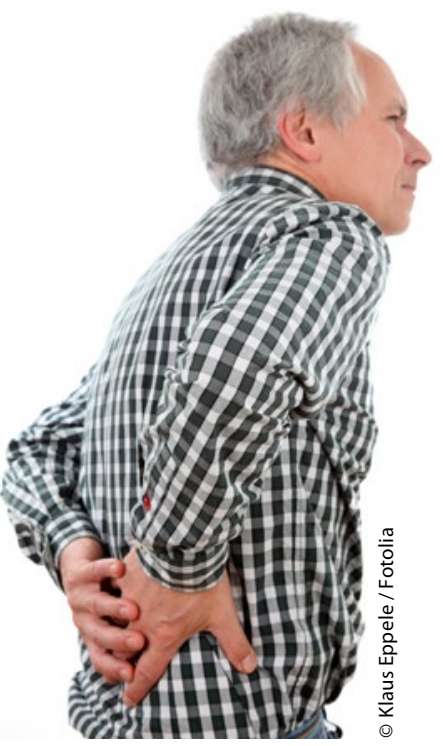

